\title{
Understanding the IT-Related Attitudes and Needs of Persons with Age-Related Macular Degeneration: A Case Study
}

\author{
Lilit Hakobyan \\ School of Engineering \& \\ Applied Science, \\ Aston University, \\ Birmingham, B4 7ET \\ hakobyl1@aston.ac.uk
}

\author{
Jo Lumsden \\ School of Engineering \& \\ Applied Science, \\ Aston University, \\ Birmingham, B4 7ET \\ j.lumsden@aston.ac.uk
}

\author{
Dympna O'Sullivan \\ School of Engineering \& \\ Applied Science, \\ Aston University, \\ Birmingham, B4 7ET \\ d.m.osullivan@aston.ac.uk
}

\author{
Hannah Bartlett \\ School of Life \& Health \\ Sciences, \\ Aston University, \\ Birmingham, B4 7ET \\ h.e.bartlett@aston.ac.uk
}

\begin{abstract}
In the UK, 20 per cent of people aged 75 years and over are living with sight loss; this percentage is expected to increase as the population ages (RNIB, 2011). Age-Related Macular Degeneration (AMD) is the UK's leading cause of severe visual impairment amongst the elderly. It accounts for $16,000 \mathrm{blind} /$ partial sight registrations per year and is the leading cause of blindness among people aged 55 years and older in western countries (Bressler, 2004). Our ultimate goal is to develop an assistive mobile application to support accurate and convenient diet data collection on which basis to then provide customised dietary advice and recommendations in order to help support individuals with AMD to mitigate their ongoing risk and retard the progression of the disease. In this paper, we focus on our knowledge elicitation activities conducted to help us achieve a deep and relevant understanding of our target user group. We report on qualitative findings from focus groups and observational studies with persons with AMD and interviews with domain experts which enable us to fully appreciate the impact that technology may have on our intended users as well as to inform the design and structure of our proposed mobile assistive application.
\end{abstract}

Keywords: User-Centred Design, Age-Related Macular Degeneration, Mobile Assistive Technology.

\section{INTRODUCTION}

Visual impairment has a significant impact on individuals' independence and quality of life, with 48 per cent of visually impaired people reported as feeling 'moderately' or 'completely' cut off from society (RNIB, 2011). Age-related macular degeneration (AMD) is the most common cause of sight loss in the UK; typically affecting people aged 50 and above, it impacts nearly one in ten of those over 80 (RNIB, 2011). As a progressive, degenerative disease of the eye it severely affects the macula, located at the centre of the retina, which is vital for clear central vision. In most cases, people with AMD have "dry" AMD, where pigment and light detection cells in the retina die off and the person experiences gradual loss of central vision (see Figure 1) but, with some people, this can progress to "wet" AMD which results in rapidly reduced central vision (RNIB, 2011).

Strategies to combat AMD are now focusing on prevention of AMD progression rather than expensive pharmaceutical treatments which are not universally effective. There is evidence that there is a link between dietary factors, AMD risk (Beatty, et al., 2001), and AMD progression (AREDS, 2001).

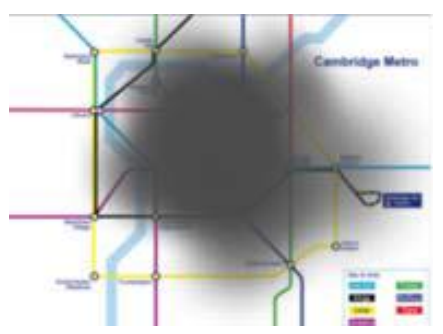

Figure 1: Example of how a subway map might be viewed through an eye affected by AMD [generated using www.inclusivedesigntoolkit.com].

In other domains - for example diabetes (Tsang, et al., 2001) - electronic diet diaries have proven to be successful aids for improving independent living. Various electronic diet diaries are available on mobile platforms - e.g. Health and Diet Manager (Softpedia, 2012) - but comprise visually-intensive Uls which are not adapted to people with visual impairment and have not been used in the specific context of dietary recommendation for AMD risk and progress mitigation. Our ultimate goal is to develop an assistive mobile application (SMART) to 
support accurate and convenient diet data collection on which basis to then provide customised dietary advice and recommendations in order to help support individuals with AMD to mitigate their ongoing risk and retard the progression of the disease. As highlighted by Figure 1, AMD presents a significant challenge in terms of UI design - a challenge which is further complicated by the degenerative nature of the disease. In recognition of this challenge, we are adopting a multidisciplinary research approach, incorporating the expertise of computer science, $\mathrm{HCl}$, ophthalmology, and psychology. Central to our approach is a user-centred, participatory design philosophy, whereby we have commenced and will continue to include target users and stakeholders (e.g. persons with AMD, clinicians, carers, etc.) throughout all stages from the design to deployment of the SMART application; we believe this to be a novel approach to IT development with this target audience. In this paper, we report on the initial, knowledge elicitation phases of our research which have been conducted using a combination of focus groups, interviews, and in-home observational studies. We hope that our findings will be of value to the wider $\mathrm{HCl}$ community in terms of better understanding the challenging ITbased needs of this target group.

\section{BACKGROUND}

\subsection{Designing for Ageing People}

$\mathrm{HCl}$-based research is increasingly exploring possibilities for enabling the ageing population to take full advantage of technological advancements. Several studies have identified the main barriers for the use of technology by older adults to be counterintuitive interfaces (Arnott, et al., 2004), unfamiliarity with computers (Czaja, et al., 2006) and physical and sensory impairments that accompany ageing (Hawthorn, 2000). Perhaps the biggest limitation of technology use by the elderly is the fact that such technologies are not typically designed to meet older adults' needs, wants and capabilities (e.g. Czaja, et al., 2006). That said, advances in the field of gerontechnology (Bouma, et al., 2007) and approaches such as universal and inclusive design (Keates, et al., 2000) are encouraging designs that better consider the needs of users such as the elderly and the disabled. The recent past has seen the emergence of specialist centres, such as Dundee University's 'User Centre', which engage older adults in research and development of technological solutions based on their needs and wants (Forbes, et al., 2009). Furthermore, the advent of mobile devices has led to increased research into making mobile devices more accessible to older adults (e.g. Leung, et al., 2010).

\subsection{Designing for the Visually Impaired}

Recent advances in the likes of vibrotactile, text-tospeech (TTS), and gestural recognition systems have also opened up scope for increased accessibility to technological devices for persons with visual impairment. Further, with the rise of mobile technologies that are incorporating touchsensitive screens, we have seen an increase in research into touchscreen accessibility for the visually impaired (e.g. Neff and Mehigan, 2010).

Technologies designed specifically for the visually impaired have the potential to enhance their quality of life via improved autonomy and safety. Advances in assistive technology for the visually impaired to sustain their independence have, for example, highlighted innovations in information accessibility (e.g. Chen, et al., 2006), navigation and wayfinding support (e.g. AbdulRasool and Sabra, 2011) and independent shopping (e.g. Kulyukin and Kutiyanawala, 2010). When designing for the visually impaired, it is important to consider the differences in individuals' visual capabilities (Jacko and Sears, 1998). In an attempt to meet the needs of users with different levels of physical/sensory impairments, researchers have developed tools for automatically generating interfaces based on users' different specifications and preferences (e.g. Nichols, et al., 2002). These tools do not, however, support UI adaptation over time based on observed (degenerating) capabilities. Designing IT for people with $A M D$ is a relatively under-researched area and, as illustrated by Figure 1, users with AMD have very specific needs and visual capabilities which degenerate over time.

\section{METHOD}

To fully understand the perspectives and appreciate the needs, difficulties, and viewpoints of individuals with $\mathrm{AMD}$, we gathered ethnographic data via a series of focus groups and observational studies with members of our target user group. We also conducted a series of interviews with AMD/clinical experts. We attended several local community support groups for people with AMD to allow us to start getting to know the community and, thereafter, to enable us to build a trusted professional relationship with individuals in an environment in which they were comfortable and to ultimately elicit their voluntary involvement in our knowledge elicitation process.

We ran focus groups every few weeks at a venue convenient to the participants (generally at the same venue as their normal support group meeting or a nearby coffee shop). From a total of 10 volunteers (nine individuals with $A M D$ and one carer), we established two working groups (where the individuals within each group knew each other 
via their respective support group), one which met three times and one which met four times over a period of 4 months. Each focus group session lasted no more than two hours and comprised five people plus the researcher/moderator. All focus group sessions were audio-recorded: a verbatim transcription of each recording was subsequently generated for analysis.

Using a brief questionnaire (appropriately formatted for the audience), we collected high level information about the AMD participants and their type of AMD, etc. Of the nine participants with $A M D$, all were over 70 years of age, with five being over the age of 80 . One participant had 'dry' AMD in one eye; two had 'dry' AMD in both eyes; all others had both 'dry' and 'wet' AMD in both eyes. The mean number of years since diagnosis for participants was 8.5 , with a range of 6 to 12 years.

In order to acquire a deeper understanding of participants' daily coping strategies and what it is like to live with AMD, we conducted a series of inhome observation sessions over the course of three months. We recruited 4 participants from the focus groups (one male and three female) who were engaged with the process and willing to participate in this additional knowledge elicitation phase. All 4 participants lived on their own. In total, six observational sessions were conducted; the number of sessions conducted per participant was determined by availability and also by a considered judgement as to whether additional sessions with the given individual would return new data. Observations of daily in-home activities were kept very informal to ensure participants felt at ease: handwritten notes were taken, and the researcher engaged in discussion with each participant as benefitted the situation.

The final (ongoing at time of writing) stage of our knowledge elicitation is taking a different approach. We are conducting one-on-one interviews with clinicians/ophthalmologists; to date, we have completed 5 interviews, with further interviews anticipated.

\section{FINDINGS}

We present the findings of our knowledge elicitation here not as definitive or exhaustive but rather as a case study rendering qualitative information and observations about the AMD user group and their attitudes towards and needs relative to technology. We hope that this information is useful to others designing for individuals with AMD.

\subsection{Focus Groups}

The first three sessions for each focus group were structured to gain insight into participants' views, perceptions on, and attitudes towards technological devices per se. Of specific note was the enthusiasm with which participants viewed current technological devices in terms of what they can offer individuals with AMD and the potential for such technology to enhance individuals' independence and quality of life.

A further, perhaps unexpected but nevertheless encouraging, discovery was the extent and breadth of participants' interpretation of 'technological devices' and/or 'technology'. Besides the most commonly referenced 'mobile phones' and 'laptop/computers', participants also included in the technology discussion ATM machines, digital TVs and radios, Kindles ${ }^{\mathrm{TM}}$, microwaves, and washing machines, amongst others. Although all participants had owned a mobile phone for an extended period of time, this technology was the least favoured by them. Participants only used mobile phones for 'phoning taxi services' and receiving and making 'urgent calls' when outdoors; mobile phones were never used for casual conversations. Participants' principal concerns or difficulties with mobile phones included overcomplicated functions, small buttons and/or screen size (hence the fear of pressing incorrect buttons), and, most importantly, lack of awareness about the available functionality on their mobile phones. Four participants agreed that the only advantage of carrying a mobile phone when outdoors was their increased sense of security. Some enthusiastically made suggestions for improving the accessibility of mobile phones - e.g.:

\footnotetext{
"I would welcome any type of sound feedback for all actions and even better if I could speak to the phone. I would like to use it for different purposes and wouldn't worry about the attention it might attract".
}

The least commonly owned device was a computer. Only three participants owned computers but their comments emphasised the key role that computers play, and the benefits they gain from using them, in their daily lives:

\footnotetext{
"It makes you feel part of the world, so you don't feel isolated. I would not be without my computer".
}

Of the three computer-literate participants only one had used Skype and YouTube. There was some evidence that participants' lacked knowledge about what features are available on mobile phones/computer - two participants suggested the following, clearly unaware of the fact such functionality was already available:

\begin{abstract}
"I would use computers if I could make letters bigger that would really help" and "I would really like a talking e-mail on my computer".
\end{abstract}

All participants found reading LCD displays, and choosing options on their microwaves, washing 
machines, etc. rather challenging and so often relied on their 'memory' and/or 'common sense'; all preferred to use old versions of the appliances because of their 'uncomplicatedness' and perhaps familiarity.

Some of the focus group sessions aimed to discover participants' coping strategies in terms of living with $A M D$, the challenges/barriers to day-today activities posed by the disease, and their perceived independence and quality of life. Shopping and safe mobility when outdoors proved immense challenges for all participants. Despite this, their views on online shopping varied: while some argued that online shopping would, in fact, limit their independence, this view was not shared by all, as illustrated by the following quote:

\section{"Online shopping makes me independent and it's fun".}

A further challenge and limit to their independence was 'exploring new places' without someone accompanying them, or 'trying out new things (e.g. sewing/knitting). Despite their limited independence and daily challenges, participants were remarkably optimistic and hopeful about their quality of life - as noted by one participant:

\section{"Independence and quality of life go hand in hand and technology could certainly fit in".}

The final focus group meetings concentrated on our proposed SMART application. During these meetings, participants had the opportunity to experiment with a few relevant applications on an iPhone and an i-Pad in order to give them a feel for the technology and current application designs and to encourage related discussion and feedback. All participants found the interaction with the i-Phone very 'challenging' and 'frustrating' due to its limited screen size, touchscreen sensitivity, and 'overloaded' interface with small icons.

In comparison, the larger i-Pad received a positive response from all participants who considered its advantages to include the 'larger screen size', 'larger icons', and the fact that it is 'lightweight' but 'portable' and 'mobile' (yet with a prospect of being used as a 'desktop computer' whenever necessary). An important issue raised by one of the participants (who also had arthritis) was that the majority of individuals with AMD also have other health concerns (often as a consequence of their age). This means that the visual element of $\mathrm{UI}$ design is not the only concern for designers: for instance, patients with arthritis are likely to experience greater difficulty with touchscreen sensitivity and, to combat this, participants suggested the use of 'pens' (i.e. styli) for input to overcome the limitations associated with touchscreens.
Participants were very keen on the concept of our proposed SMART application. For it to yield positive outcomes, participants suggested consideration of the following: a 'detailed instructions booklet'; 'color-coded buttons/icons'; white foreground colour on black background; the 'possibility for customisation (e.g. increase font size)'; and 'speech input'. Encouragingly, our participants had a positive and inclusive reaction to our proposed application - for example:

"It could become my best friend. It would become my companion, give me an incentive and fill my day. [...] I am gobsmacked to what technology can do and that I can be part of it".

\subsection{In-Home Observational Studies}

We noted a number of prominent trends or commonalities across all observed participants in terms of their living arrangements with respect to accommodating their visual deficiencies. These included having well-lit and simple interior design, light-coloured walls, a lot of lighting in every room, coloured 'bumps' on most appliances and switches, and preferably no stairs (only one participant was living in a house with stairs and found them very challenging, especially going down the stairs, as everything appeared in "2D" (i.e. 'flat')). Furthermore, participants typically kept their homes very tidy, organised, and, most importantly, kept things 'handy'. For example, all participants had a small table or chair in their living room on which they kept 'everyday things' such as medication, razors, remote controllers, glasses, magnifiers, and emergency contact numbers. In their kitchens, all jars, cans and bottles were organised and out on display to eliminate the need for looking into cupboards and enable them to see/find things more easily. Participants were generally inclined to put things back where they belonged (in the same place every time) so they knew without thinking where to find things. Being organised was generally considered crucial for maintaining an independent life. The main difficulties encountered by participants at home included losing things and/or friends/relatives misplacing things when trying to help.

Three participants were observed whilst preparing food in the kitchen. Surprisingly, no notable differences were observed between their working methods and that of a sighted person; one participant did, however, note the challenge associated with 'seeing' what was on a plate when eating.

In terms of observed used of technology in the home, 2 participants owned computers and used them primarily for e-mailing and 'researching'. In both cases, the participants' e-mail accounts were constantly logged in and were one-click away via the desktop. We observed considerable differences 
between their approach to interaction with a desktop computer and with portable devices/objects: while the desktop required no 'special viewing' technique (participants were sitting slightly closer to the screen and looking 'straight' at it), both participants had to hold portable devices/objects (e.g. Kindle ${ }^{\mathrm{TM}}$, book) slightly to their left side and under a lamp to be able to read. Participants also preferred yellow font on black background when reading digital books, newspapers and magazines.

\subsection{Interviews with AMD/Clinical Experts}

Domain experts (e.g. clinicians, ophthalmologists) are currently being consulted to elicit their expert opinion on how to design our proposed technology such that it can best fit into the lives of individuals with AMD and, most importantly, benefit their lives. Although not yet complete, the main information/suggestions emerging from our interviews to date include:

(i) the importance of making the effect/outcome of our proposed solution 'perfectly clear' to patients/participants to mitigate against misunderstandings as a result of mix-matched expectations;

(ii) the need for taking into account any vitamins/supplements patients might take and/or possibility of other age-related impairment;

(iii) information about the effect nutrition can have on different levels of visual impairments; and

(iv) that 'simplicity' and 'speech input/output' are key for successful use of our proposed device or any assistive technology in general.

\section{DISCUSSION}

Due to the heterogeneity of capabilities of individuals with AMD, multimodality and maximal flexibility should be a priority so that users can personalise the systems to their individual needs and capabilities and taking into account their rate of degeneration. Our studies suggest that individuals with AMD need more time to locate and identify things on screen which stresses the importance of providing the ability to customise aspects of the user interface such as optimal font and icon size and extended timeouts to support effective identification of visual content. As already mentioned, besides their visual deficiencies people with AMD are likely to have other age-related impairments: whilst, as a consequence of lack of knowledge about technological advances, participants only identified speech recognition technology as an essential requirement for their user group, we would argue that multimodality in general is significant to not only compensate for visual deficiencies but to also accommodate comorbidity issues.

Our findings indicate that an important consideration when designing for people with AMD is the environment where technology is going to be used; our observational studies showed that lighting levels and distance from screen can greatly influence the perceived usability of a device. Thus, design should accommodate different viewing distances rather than assume a one-size-fits-all solution. Contrast sensitivity is significant when designing for people with AMD (as testified to in large part by the colour schemes within their homes): participants' comments advocated the need for 'white' and/or 'yellow' foreground (i.e. icons, buttons, font) on 'black' background. Our findings further suggest that usability could be enhanced by avoiding unnecessarily colourful displays yet, conversely, 'carefully' colour-coding high-contrast buttons and icons: the black 'circle' at the centre of their vision makes it impossible to differentiate dark/similar colours close to each other or on top of one another. Despite advances in making mobile phones more accessible for the visually impaired, we would argue that such devices are not yet fully accessible to people with AMD. In particular, the size of the display is a key limiting factor for this user group; the loss of central vision makes it very difficult to see fine details, especially important details placed in the centre of the display, with the result that they find the 'small' screens of mobile phones rather challenging. We identified an imperative need for intuitive and consistent design so that participants can rely on their memory if and when necessary. A dependence on memory and consistent layout of their living environments was noted as being of utmost importance to this user group and this finding should translate to any technology design; although consistent design, layout and navigation are important elements of any user interface design, they are a vital necessity for this user group. This also has obvious implications for any automated UI layout adaptation algorithm we develop to accommodate their degenerating capabilities. Based on our findings, we stress the importance of training users in the use of the technology. Talking to and observing our participants has highlighted the fact that comprehensive instructions on setting up and using any form of technology can help users become familiar with the equipment and its available functionality, and also to understand how it can fit into their daily routine. This can further help in minimising fear of making an error - our findings revealed that this is one of the main reasons individuals with AMD are reluctant to use new devices. As a result of the degenerative nature of the disease, changes in visual capabilities will 
adversely and changeably affect individuals' interaction with technology over time. Thus, the design of the UI must be capable of making allowances for trial-and-error and of identifying and adapting to users' vision changes over time. In the next phase of our project, we also plan to examine, in more depth, how individuals' visual acuity affects their interaction style. During our observations it was noted that participants positioned books and other devices (e.g. Kindles ${ }^{\mathrm{TM}}$ ) to maximise vision via the 'better' eye. Based on this, we believe the 'better' eye (i.e. the one not affected by AMD or the one with better peripheral vision) to be an important consideration for UI layout. Although it needs to be confirmed via appropriate optometry examinations, we surmise from our observations that a weighted average acuity from both eyes will impact on an AMD user's interaction style.

\section{CONCLUSIONS AND FUTURE WORK}

This work, whilst representing the initial knowledge elicitation stages of an ongoing research agenda, contributes qualitative and previously unstudied information about the attitudes, needs, wants and capabilities of individuals with AMD as they relate to information technology. It is our intention to incorporate this understanding into the design of our SMART application via a multidisciplinary and participatory design approach. We hope, however, that this information is of value to others faced with the challenge of designing technology for an AMD target group.

\section{REFERENCES}

AbdulRasool,D. \& Sabra,S. (2011) MobileEmbedded Smart Guide for the Blind. In: DICTAP 2011, Dijon, France, June 21-23, 571- 578.

Arnott, J.L., et al. (2004) Email interfaces for older people, In: IEEE International Conference on Systems, Man \& Cybernetics, Hague, Netherlands, October 10-13, 111-117.

Beatty, S., et al. (2001) Macular Pigment and Risk for Age-Related Macular Degeneration in Subjects from a Northern European Population. Investigative Ophthalmology \& Visual Science, 42(2), 439-446.

Bouma, H., et al. (2007) Gerontechnology in perspective:Review. Gerontech. Journal, 6(4), 190216.

Bressler, N.M. (2004) Age-related macular degeneration is the leading cause of blindness. JAMA, 291, 1900-1901.

Chen, X., et al. (2006) AudioBrowser: a Mobile Browsable Information Access for the Visually Impaired. Universal Access in the Information Society, 5(1), 4-22.
Czaja, S., et al. (2006) Factors predicting the use of technology: findings from (create). Psychol Aging, 21(2), 333-352.

Forbes, P., et al. (2009) Dundee user centre: a space where older people and technology meet. In: SIGACCESS, Orlando, Florida, USA, October 2529, 231-232.

Hawthorn, D. (2000) Possible implications of aging for interface designers. Interacting with Computers, 12, 507-528.

Jacko, J.A. and Sears, A. (1998) Designing interfaces for an overlooked user group: Considering the visual profiles of partially sighted users. In: ASSETS '98, Marina Del Rey, CA, USA, April 15-17, 75-77.

Keates, S., et al. (2000) Towards a practical inclusive design approach. In: CUU'00, Arlington, VA, USA, November 16-17, 45-52.

Kulyukin, V., and Kutiyanawala, A. (2010) Accessible Shopping Systems for Blind and Visually Impaired Individuals. The Open Rehabilitation J., 3, 158-68.

Leung, R., et al. (2010) Multi-Layered Interfaces to Improve Older Adults' Initial learnability of Mobile Applications. ACM Transactions on Accessible Computing, 3(1), 1-5.

Neff, F. and Mehigan, T.J. (2010) Accelerometer \& spatial audio technology: Making touch-screen

mobile devices accessible. In: ICCHP, Vienna, Austria, July, 2010, 170-177.

Nichols, J., et al. (2002) Generating remote control interfaces for complex appliances. In: UIST'02, Paris, France, October 27-30,161-170.

Phillips, B. and Zhao, H. (1993) Predictors of assistive technology abandonment. Assistive Technology, 5(1), 36-45.

RNIB, (2010) Key Information and Statistics. http://www.rnib.org.uk/aboutus/research/statistics/P ages/statistics.aspx (12 December 2011).

Softpedia (2012) Personal Health \& Diet Manager. http://handheld.softpedia.com/get/Health/Nutrition/

Personal-Health-Diet-Manager-4488.shtml June 2012).

The AREDS Research Group, (2001) Archives of Ophthalmology, 119(10), 1417-1436.

Tsang, et al. (2001) Improvement in diabetes control with a monitoring system based on a handheld, touch-screen electronic diary. $J$ Telemed Telecare, 7, 47- 50 . 